\title{
Billiard knots in a cylinder
}

\author{
C. Lamm, D. Obermeyer \\ Mathematisches Institut, Beringstr. 1, \\ 53115 Bonn, Germany, \\ e-mail: lamm@math.uni-bonn.de
}

\begin{abstract}
We define cylinder knots as billiard knots in a cylinder. We present a necessary condition for cylinder knots: after dividing cylinder knots by possible rotational symmetries we obtain ribbon knots. We obtain an upper bound for the number of cylinder knots with two fixed parameters (out of three). In addition we prove that rosette knots are cylinder knots.
\end{abstract}

\section{Introduction to billiard knots}

Billiard knots were introduced in the articles [4] and [8]. They are periodic billiard trajectories without self-intersections in some billiard room in $\mathbb{R}^{3}$. One case mentioned by V. Jones and J. Przytycki in [4] seems especially interesting to us: the case of billiard knots in a cylinder. In this article we derive a necessary condition for cylinder knots which shows that not all knots can be realized by them. First of all, we give a formal definition of billiard knots.

Definition 1.1 Let $M$ be a 3-manifold in $\mathbb{R}^{3}$ with piecewise smooth boundary $\partial M$. The knot $K \subset \mathbb{R}^{3}$ is a billiard knot in $M$ if it is a polygon with

i) $K \subset M$,

ii) $\{$ vertices of $K\} \subset\{p \in \partial M \mid \partial M$ is smooth at $p\}$,

iii) at the vertices $v$ we have a reflection at the tangent plane $T_{v}(\partial M)$ as known from light-rays or billiard balls.

Also, we define a billiard link in $M$ as a collection of non-intersecting billiard knots in $M$.

Examples 1.2 1.) $M=\mathbf{D}^{3}$ : billiard knots in a standard 3-ball. Billiard curves lie in a plane and hence we only get trivial knots. However non-trivial links like the Hopf links 
are obtainable. Every component of such a link is trivial and the linking number of two of them is $-1,0$ or 1 . It is as yet unknown which links exactly occur in the standard ball.

2.) $M=\mathbf{I}^{3}$ (cube). As explained in [4] and [8] billiard knots in a cube are the same as Lissajous knots defined in [1]. (Recently we have found that the relationship between Lissajous curves and billiard curves in cube was already well known to mathematicians who study billiards, see for instance [2], p. 294, and [3]). The Alexander polynomial of a Lissajous knot is a square modulo 2; from this we concluded that its Arf invariant vanishes and that algebraic knots (e.g. torus knots) are not Lissajous knots [8].

\section{Elementary properties of cylinder knots}

Definition 2.1 A cylinder knot is a billiard knot in $\mathbf{D}^{2} \times \mathbf{I}=\{(z, x) \in \mathbb{C} \times \mathbb{R}:|z| \leq$ $1,0 \leq x \leq 1\}$

We project the billiard curve on $\mathbf{D}^{2}$ and consider one edge of the projection, see Figure 1. Together with the center $M(=0 \in \mathbb{C})$ of $\mathbf{D}^{2}$ this edge spans a triangle; let $\gamma$ be the angle at $M$. Taking the edge as a part of the billiard curve, a closed curve is achieved if and only if $\gamma=2 \pi \frac{s}{n}(s, n \in \mathbb{N})$; here and for the rest of the article we assume $\operatorname{gcd}(s, n)=1$ and $n \geq 2 s+1$. The natural number $s$ and $n$ are the rotation number and the number of reflections of the projected curve, respectively.

Let $f_{1,2}:[0,1] \rightarrow \mathbb{C}$ parametrize the projection proportional to arclength and let $f_{1,2}(0)=1 \in \mathbb{C}$ and $f_{1,2}\left(\frac{1}{n}\right)=e^{2 \pi i \frac{s}{n}} \in \mathbb{C}$ be the first and second vertices. Then the vertices of the closed billiard curve are $f_{1,2}\left(\frac{k}{n}\right)=e^{2 \pi i \frac{s}{n} k}, k=0, \ldots, n-1$.

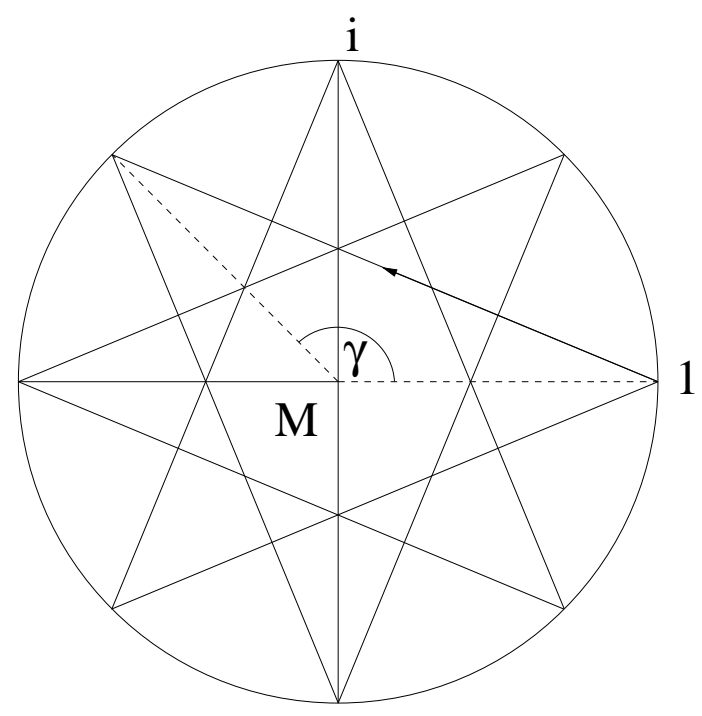

Figure 1: The projected billiard curve for $n=8$ and $s=3$.

A maximum is a point where $K$ reflects off the ceiling. The vertical movement is given by $m$, the number of maxima, and a phase $\phi$ which determines the placement of the maxima. If $g$ is the sawtooth-function $g(t)=2\left|t-\lfloor t\rfloor-\frac{1}{2}\right|$ we can write the height-function 
of the billiard curve as $f_{3}(t)=g(m t+\phi)$. We denote the resulting billiard curve (not the knot type) by $Z(s, n, m, \phi)$.

Example 2.2 If $q \geq 2 p+1$ the torus knots $t(p, q)$ are cylinder knots 沟. They are realized by $Z(p, q, q)$.

In the last line we neglected the phase on purpose, because surprisingly cylinder knots are essentially independent of it:

Lemma 2.3 Cylinder knots are independent of the phase (up to taking mirror image).

Proof: We start from a phase which gives a cylinder knot without self-intersections, and we show that by pushing the maxima along the knot, we do not change the knot up to taking mirror image.

Let us push one maximum into the direction of the knot's orientation. The knot does not change until, for the first time, the maximum reaches a point $A$ for which a singularity occurs at a crossing point $P \in D^{2}$. Let $t_{1}, t_{2} \in[0,1]$ be the parameters of $P$; hence $f_{1,2}\left(t_{1}\right)=f_{1,2}\left(t_{2}\right)=P, f_{3}\left(t_{1}\right)=f_{3}\left(t_{2}\right)$. By the symmetry of the function $g$ we have $g\left(m t_{1}+\phi\right)=g\left(m t_{2}+\phi\right) \Leftrightarrow m\left(t_{1}-t_{2}\right) \in \mathbb{Z}$ or $m\left(t_{1}+t_{2}\right)+2 \phi \in \mathbb{Z}$. If $m\left(t_{1}-t_{2}\right) \in \mathbb{Z}$ then the singularity cannot be removed by changing $\phi$. If $m\left(t_{1}+t_{2}\right)+2 \phi \in \mathbb{Z}$ then a sufficiently small change of $\phi$ removes the singularity. These two cases correspond to a) and b) in Figure 2, respectively. Now a) implies there was already a singularity at $P$ before pushing the maximum along the knot. Hence $P$ must look like b). We have for all $\delta \in[0,1]$ the equation $f_{3}\left(t_{1}+\delta\right)=f_{3}\left(t_{2}-\delta\right)$. Therefore the knot is symmetric with respect to the plane spanned by $P$ and the central axis of $\mathbf{D}^{2} \times \mathbf{I}$. By $A^{\prime}$ we denote the mirror image of the maximum at $A$. The symmetry implies that there is a maximum at $A^{\prime}$ as well. If now we continue pushing the maximum at $A$ along the knot, from $A^{\prime}$ we push the other maximum into the mirror image of the region we came from (see Figure 2 $\mathrm{c}$ ), and we get the mirror image of the knot we started from.

a)

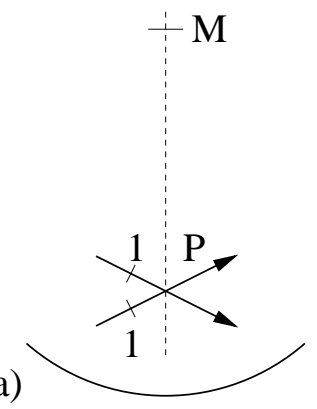

b)

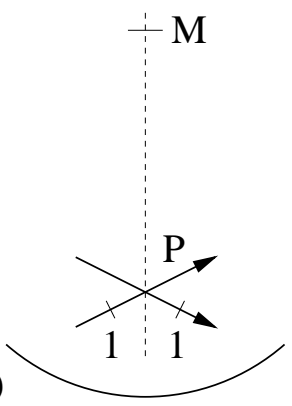

c)

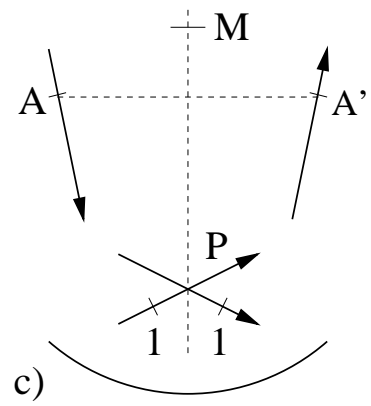

Figure 2: a) A non-removable singularity, b) a removable singularity, c) global symmetry in case b)

As already mentioned the configuration of Figure 2a) leads to a self-intersection at $P$ for every phase. However we can conclude that it does not occur by using the following lemma from number theory. 
Lemma 2.4 If $\alpha, \beta \in \mathbb{Q}$ with $0<\beta<\alpha<\frac{1}{2}$ satisfy $\tan (\pi \alpha)=\lambda \tan (\pi \beta)$ for some $\lambda \in \mathbb{Q}$, then $\alpha=\frac{1}{3}, \beta=\frac{1}{6}, \lambda=3$.

Proof: $\quad$ Let $\alpha=\frac{p_{1}}{q_{1}}, \beta=\frac{p_{2}}{q_{2}}$ with $\operatorname{gcd}\left(p_{1}, q_{1}\right)=\operatorname{gcd}\left(p_{2}, q_{2}\right)=1$ and $\tilde{\alpha}=e^{2 \pi i \alpha}, \tilde{\beta}=e^{2 \pi i \beta}$. Then $\frac{\tilde{\alpha}-1}{\tilde{\alpha}+1}=i \tan (\pi \alpha), \frac{\tilde{\beta}-1}{\tilde{\beta}+1}=i \tan (\pi \beta)$. Hence the cyclotomic fields $\mathbb{Q}[\tilde{\alpha}]$ and $\mathbb{Q}[\tilde{\beta}]$ are equal. This is possible only in the two following cases.

First case: $q_{1}=q_{2}$. This is a contradiction to the fact that $\cot (\pi \alpha)$ and $\cot (\pi \beta)$ are rationally independent by [12].

Second case: $q_{2}=2 q_{1}, q_{1}$ odd. Then $-\tilde{\beta}$ is conjugate to $\tilde{\alpha}$ and $\frac{-\tilde{\beta}-1}{-\tilde{\beta}+1}$ is conjugate to $\frac{\tilde{\alpha}-1}{\tilde{\alpha}+1}$.

The degree of the extension is $\varphi\left(q_{1}\right)$ and we find that the norm of $\frac{\tilde{\alpha}-1}{\tilde{\alpha}+1}$ is $\lambda^{\frac{\varphi\left(q_{1}\right)}{2}}$ because $\frac{-\tilde{\beta}-1}{-\tilde{\beta}+1} \frac{\tilde{\alpha}-1}{\tilde{\alpha}+1}=\left(1 / \frac{\tilde{\beta}-1}{\beta+1}\right) \cdot \frac{\tilde{\alpha}-1}{\tilde{\alpha}+1}=\lambda$. But we know that this norm is either 1 or a prime number, because $\tilde{\alpha}-1$ generates a prime ideal in the ring of integers of $\mathbb{Q}[\tilde{\alpha}]$ if $q_{1}$ is a prime-power and it is a unit otherwise, see [13]. Hence $\frac{\varphi\left(q_{1}\right)}{2}=1$ and this implies $q_{1}=3$ because 3 is the only odd number $q$ with $\varphi(q)=2$.

Proposition 2.5 In a billiard curve in the cylinder $Z(s, n, m, \phi)$ every singularity is removable by changing $\phi$.

Proof: We need to show that if $t_{1}$ and $t_{2}$ are the parameters at a crossing $P_{b}(0<b<s)$ then there is no $m$ so that $m\left(t_{1}-t_{2}\right) \in \mathbb{Z}$, i.e. that $t_{1}-t_{2} \in \mathbb{Q}$. We scale the projection so that its total length is 1 . Using the triangle $M P_{0} P_{s}$ we get $\left|M P_{0}\right|=1 /\left(2 n \tan \left(\frac{\pi s}{n}\right)\right)$ because $\left|P_{0} P_{s}\right|=\frac{1}{2 n}$, and from the triangle $M P_{0} P_{b}$ we compute $\left|P_{0} P_{b}\right|=\frac{1}{2 n} \frac{\tan \left(\frac{\pi b}{n}\right)}{\tan \left(\frac{\pi s}{n}\right)}$.

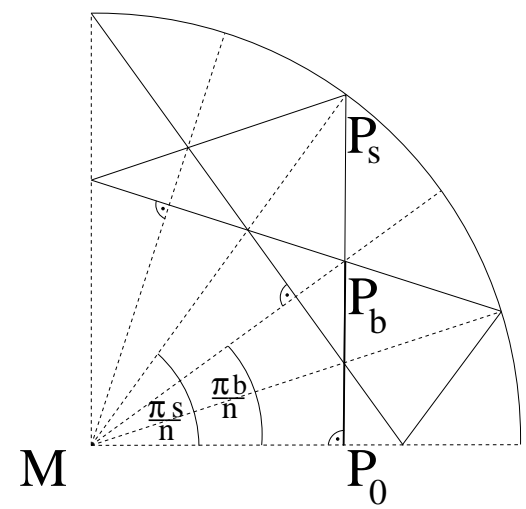

So there are $k_{1}, k_{2} \in \mathbb{Z}$ with

$$
t_{1}=\frac{k_{1}}{2 n}+\frac{1}{2 n} \frac{\tan \left(\frac{\pi b}{n}\right)}{\tan \left(\frac{\pi s}{n}\right)}, t_{2}=\frac{k_{2}}{2 n}-\frac{1}{2 n} \frac{\tan \left(\frac{\pi b}{n}\right)}{\tan \left(\frac{\pi s}{n}\right)} .
$$

From this it is immediate that if $m\left(t_{1}-t_{2}\right) \in \mathbb{Z}$ then

$$
\tan \left(\frac{\pi b}{n}\right) / \tan \left(\frac{\pi s}{n}\right) \in \mathbb{Q}
$$

Lemma 2.4 shows that the only solution is $n=6 b, s=2 b$ which contradicts $\operatorname{gcd}(s, n)=1$.

From now on we will denote a cylinder knot by $Z(s, n, m)$ but keep in mind that the knot-type is determined by this notation only up to mirror image. However, the billiard curve is denoted by $Z(s, n, m, \phi)$. 
We take a look at the symmetries of cylinder knots (compare [ [4], Theorem 3.11). A knot $K$ has cyclic period $n$ if $K$ is fixed under a rotation of $\frac{2 \pi}{n}$ around an axis $h$ with $h \cap K=\emptyset$. The linking number associated to this rotation is $l k(h, K)$. A knot $K$ is strongly positive amphicheiral if there is an involution $i: \mathbb{R}^{3} \rightarrow \mathbb{R}^{3}$ with $i(K)=K$, preserving the orientation of the knot and reversing the orientation of $\mathbb{R}^{3}$.

Lemma 2.6 a) If $\operatorname{gcd}(n, m)=d>1$, then $Z(s, n, m)$ has cyclic period $d$ with linking number s. Conversely, if $Z(s, n, m, \phi)$ has rotational symmetry $d^{\prime}$ then $d^{\prime} \mid d$.

b) If $n$ is even and $m$ is odd, then $Z(s, n, m)$ is strongly positive amphicheiral.

Proof: a) A rotation of $\frac{2 \pi}{n}$ about the central axis corresponds to $t \mapsto t+\frac{k}{n}$ in the parametrization, where $k s \equiv 1(\bmod n)$. Hence for $d \mid n$ the shift $t \mapsto t+\frac{k}{d}$ gives a rotation of $\frac{2 \pi}{d}$, which maps the projection to itself. But because $d \mid m$ we have $f_{3}(t)=f_{3}\left(t+\frac{k}{d}\right)$; this shows that the curve $Z(s, n, m, \phi)$ is rotationally symmetric with period $d$. For the converse let $d^{\prime} \in \mathbb{N}$ be a rotational symmetry of the diagram. Then for the projection we need $d^{\prime} \mid n$ and for the height function $\frac{1}{d^{\prime}} \in \frac{1}{m} \mathbb{Z}$. Then $d^{\prime} \mid m$ and hence $d^{\prime} \mid d$.

In the case of b) a rotation of $\pi$ preserves the diagram but exchanges maxima and minima: the curve is symmetric with respect to $\left(0, \frac{1}{2}\right)$, the center of the cylinder.

The number $d$ is the maximal period of the diagram. We call it the maximal billiard period.

Remark 2.7 In the periodic case the factor $k n o t K^{(d)}$ is obtained by identifying the radial faces of a $\frac{2 \pi}{d}$-slice of the cylinder. We do this by a process of enlarging the slice to the whole cylinder, that is (in cylinder coordinates) mapping the angle $\alpha$ to $\alpha \cdot d$. The result is no longer a billiard knot since its lines are not straight but it still has those symmetries of cylinder knots which are derived from the dihedral symmetries of their projections.

\section{A necessary condition for cylinder knots}

In this section we prove that not every knot can be a cylinder knot. In order to show this, we will prove that after factoring by the maximal billiard period, the resulting factor knot is a ribbon knot.

We want to allow maxima and minima on the boundary of $\mathbf{D}^{2}$ as well, although our definition of billiard knots forbids this because the boundary of $\mathbf{D}^{2} \times \mathbf{I}$ is not smooth there. Instead we start from the projected curve and distribute the maxima equidistantly.

Lemma 3.1 a) A maximum or minimum at a vertex $P$ on the boundary of $\mathbf{D}^{2}$ yields singularities at every crossing point on the plane spanned by $P$ and the central axis of $\mathbf{D}^{2} \times \mathbf{I}$.

b) If $\operatorname{gcd}(n, m)=1$ then there are no singular crossings other than the singularities mentioned in a).

Proof: a) The knot projection on $\mathbf{D}^{2}$ is symmetric with respect to the plane spanned by $P$ and the central axis $0 \times \mathbf{I}$. Now if we have a maximum or minimum at $P$ then the height function is symmetric as well, so the whole billiard curve is symmetric with respect to the plane. This can only happen if the crossing points on the plane are self-intersections. 
b) Similarly to a), a singularity implies (Figure 2, Proposition 2.5) that the billiard curve is symmetric with respect to the plane spanned by the crossing point and the central axis. Hence a singular crossing outside the symmetry plane of a) yields a second symmetry plane of the billiard curve. The product of these two reflections is a rotation around the central axis fixing the curve. But since $\operatorname{gcd}(n, m)=1$ this is impossible (Lemma 2.6).

A ribbon knot is a knot which is the boundary of a singular disk with only ribbon singularities.

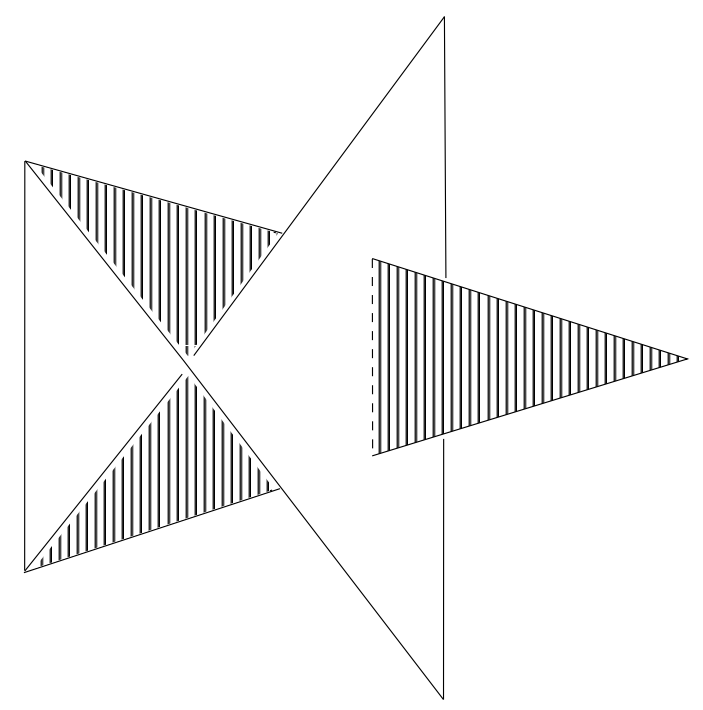

Figure 3: A ribbon for a cylinder knot diagram

Proposition 3.2 Let $K=Z(s, n, m)$ be a cylinder knot and $d=\operatorname{gcd}(n, m)$ its maximal billiard period. Then the factor knot $K^{(d)}$ is a ribbon knot.

Proof: Remember that $K^{(d)}$ still admits the symmetries of cylinder knots (Remark 2.7). The idea to prove this proposition is to connect points with their mirror image as $A, A^{\prime}$ in Figure 2c). This is similar to the well-known technique for $K \sharp\left(-K^{*}\right)$.

Let $f_{1,2}:[0,1] \rightarrow \mathbf{D}^{2} \subset \mathbb{C}$ be the mapping describing the projection of $K^{(d)}$ with $f_{1,2}(0)=f_{1,2}(1)=1$, and $f_{3}:[0,1] \rightarrow \mathbf{I}$ the height function starting with a maximum at 1: $f_{3}(1)=1$. By the preceding lemma we get singularities on $\mathbb{R} \times \mathbf{I}$ and there are no further singularities.

Now by the following steps we construct a ribbon for $K^{(d)}$ : In step 4) the singularities on $\mathbb{R} \times \mathbf{I}$ are removed so that they unfold to half-twists in the ribbon.

1) We start with a mapping

$$
\begin{aligned}
B:[0,1]^{2} & \rightarrow \mathbf{D}^{2} \times \mathbf{I} \\
(x, y) & \mapsto\left((1-y) f_{1,2}\left(\frac{x}{2}\right)+\overline{f_{1,2}\left(\frac{x}{2}\right)}, f_{3}\left(\frac{x}{2}\right)\right) .
\end{aligned}
$$


Let $S=\operatorname{im}(B)$ be the singular disk described by $B . B(x, 0)$ parametrizes the first half of $K^{(d)}$; the second half is parametrized by $B(x, 1)$ since $K^{(d)}$ is symmetric with respect to $\mathbb{R} \times \mathbf{I}$. $B(0, y)$ and $B(1, y)$ are constant. Hence $K^{(d)}$ is the boundary of $S$.

2) Because of the symmetry of $B$, all the self-intersections of $S$ are ribbon singularities.

3 ) If three or more parts of the ribbon meet in one arc, we may deform $S$ in a sufficiently small neighbourhood to push away one of the parts without destroying the symmetry.

4) Now let us finally push away the maximum from 1 to remove the singularities on $\mathbb{R} \times \mathbf{I}$. One of the two sides will yield the desired knot (the other its mirror image, see the proof of Lemma 2.3). As long as the maximum is sufficiently close to 1 , we do not change the knot outside $\mathbb{R} \times \mathbf{I}$ (by Lemma 3.1, b)), and we move $S$ together with the knot. The singularities on $\mathbb{R} \times \mathbf{I}$ open to form half-twists in $S$, and the result is the desired ribbon for our factor knot (Figure 3).

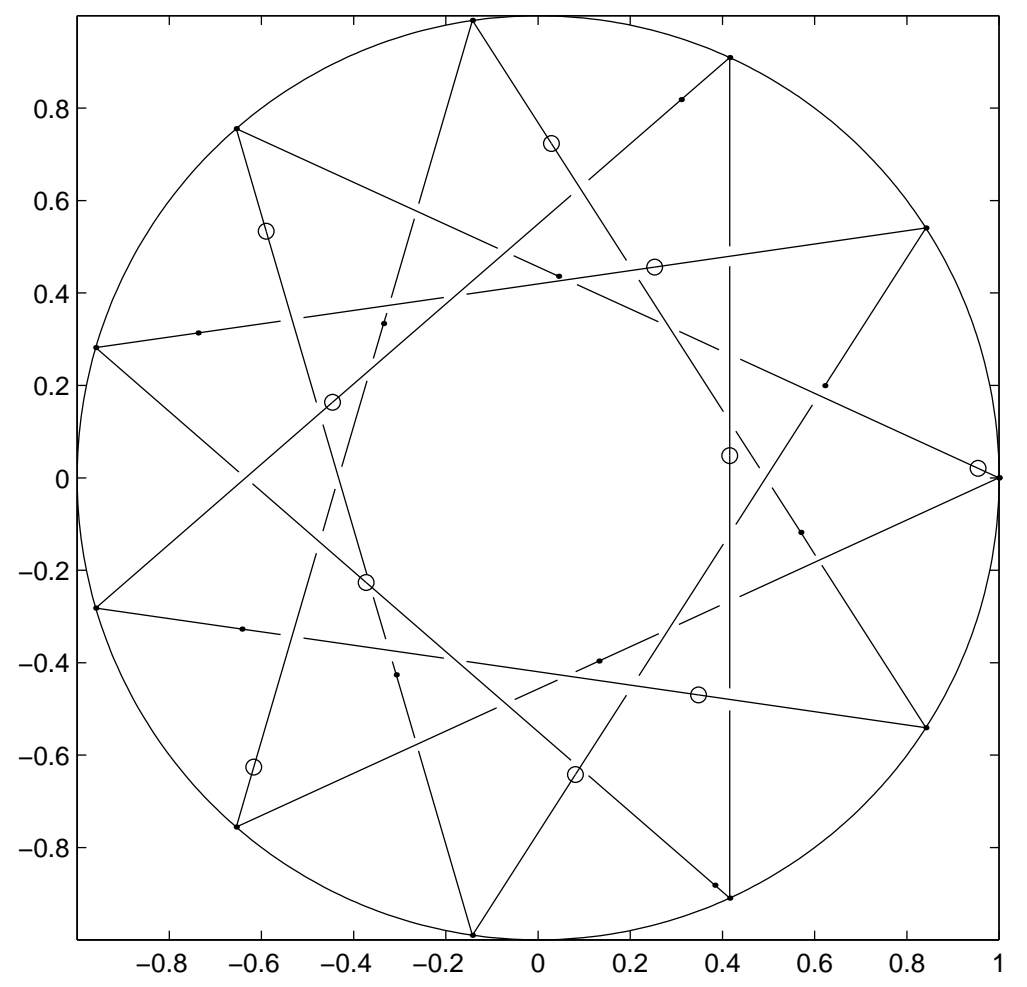

Figure 4: An example of a ribbon cylinder knot: $Z(4,11,10)$. The circles and dots are the positions of the maxima and minima.

Remark: We observe that $K^{(d)}$ is a generalized union of a knot diagram and its mirror image as defined in [6] and [9].

We sum up what we know about cylinder knots:

Theorem 3.3 Let $K=Z(s, n, m)$ be a cylinder knot and $d=\operatorname{gcd}(n, m) \quad(d=1$ may happen). Then $K$ has cyclic period $d$ with linking number $s$ and the factor knot $K^{(d)}$ is ribbon. In particular, $K^{(d)}$ satisfies: 
a) The determinant is a square,

b) the Arf invariant vanishes,

c) the signature vanishes.

If $\frac{n}{d}$ is even then $K^{(d)}$ is also strongly positive amphicheiral.

Proof: By Proposition $3.2 K^{(d)}$ is a ribbon knot. We know from [5] that its signature vanishes and the Alexander polynomial has the form

$$
\Delta_{K^{(d)}}(t)=F(t) F\left(t^{-1}\right)
$$

for some $F \in \mathbb{Z}[t]$. Since $\operatorname{det}\left(K^{(d)}\right)=\Delta_{K^{(d)}}(-1)$ we have a) and c). Part b) is a corollary from a) because 1 is the only odd square modulo 8 , hence $\operatorname{det}\left(K^{(d)}\right) \equiv \pm 1(\bmod 8)$ and the Arf invariant vanishes.

The diagram of $K^{(d)}$ has $\frac{n}{d}$ vertices and $\frac{m}{d}$ maxima. The numbers $\frac{n}{d}$ and $\frac{m}{d}$ are coprime; in particular, if $\frac{n}{d}$ is even then $\frac{m}{d}$ has to be odd, hence by Lemma $2.6 K^{(d)}$ is strongly positive amphicheiral.

Corollary 3.4 Cylinder knots are either periodic or ribbon. Hence not all knots are cylinder knots.

For instance, the knot $8_{10}$ (notation as in [5]) has no period and its determinant is 27 , hence it does not match our necessary condition. But not even all periodic knots are cylinder knots. The knot $5_{2}$ has 2 as its only period, but the linking number with its axis of rotation is \pm 1 ; furthermore, $\operatorname{det}\left(5_{2}\right)=7$, so $5_{2}$ cannot be a cylinder knot. Note that $5_{2}$ is the first nontrivial Lissajous knot. In fact, we know only one example of a Lissajous knot which is at the same time a cylinder knot: this is $3_{1} \sharp 3_{1}^{*}=Z(3,11,4)$.

The argument of the last paragraph shows:

Corollary 3.5 Let $K$ be a knot which is not ribbon. If the complete list of its cyclic periods together with the linking numbers with the respective axis of rotation is $\left(\left(q_{1}, \lambda_{1}\right), \ldots,\left(q_{k}, \lambda_{k}\right)\right)$ and if for all $i$ we have $\left|\lambda_{i}\right|<\operatorname{br}(K)$ then $K$ is not a cylinder knot.

\section{An upper bound for the number of cylinder knots with given $s$ and $n$}

If $Z(s, n, m)$ is a cylinder knot we write

$$
Z(s, n, m)=\prod_{i=1}^{n}\left[\prod_{\substack{j \text { odd } \\ j<s}} \sigma_{j}^{\varepsilon_{i, j}} \prod_{\substack{j \text { even } \\ j<s}} \sigma_{j}^{\varepsilon_{i, j}}\right]^{\wedge}, \varepsilon_{i, j} \in\{ \pm 1\},
$$

reading the braid in counterclockwise direction. Of course there is a choice of rotating and mirroring the braid, but for Theorem 4.1 we fix one of the braids. We consider the first subscript $i$ of $\varepsilon_{i, j}$ (and $x_{i, j}, y_{i, j}$ in the proof of Theorem 4.1) to be in $\mathbb{Z} / n \mathbb{Z}$.

If $n$ and $m$ are coprime, Theorem 4.1 says that for each $j$ we have a series of positive and negative crossings. These series have the same length (for even $n$ ), or their lengths differ only by one. 
Theorem 4.1 Let $\operatorname{gcd}(n, m)=1$ and $a \in\{1, \ldots, n-1\}$ with am $\equiv s \quad(\bmod n)$. Then for each $j \in\{1, \ldots, s-1\}$ there is exactly one $b_{j} \in\{1, \ldots, n\}$ so that for $n$ even

$$
\varepsilon_{b_{j}+i \cdot a, j}= \begin{cases}+1 & \text { for } i=0, \ldots, \frac{n}{2}-1 \\ -1 & \text { for } i=\frac{n}{2}, \ldots, n-1,\end{cases}
$$

and for $n$ odd

$$
\varepsilon_{b_{j}+i \cdot a, j}=\left\{\begin{array} { l l } 
{ + 1 } & { \text { for } i = 0 , \ldots , \frac { n - 1 } { 2 } , } \\
{ - 1 } & { \text { for } i = \frac { n + 1 } { 2 } , \ldots , n - 1 , }
\end{array} \text { or } \left\{\begin{array}{ll}
-1 \quad \text { for } i=0, \ldots, \frac{n-1}{2} \\
+1 \quad \text { for } i=\frac{n+1}{2}, \ldots, n-1
\end{array}\right.\right.
$$

Proof: We denote the crossing corresponding to $\sigma_{j}^{\varepsilon_{i, j}}$ by $(i, j)$ and the parameters of the crossing $(i, j)$ by $x_{i, j}$ and $y_{i, j}$. We assume that the $x_{i, j}$ belong to the left string at a crossing and the $y_{i, j}$ to the right string, respectively. For the proof of the theorem we fix one $j$ and omit it from the subscripts for simplification. Let $k \in\{1, \ldots, n-1\}$ as in the proof of Lemma 2.6 satisfy $k s \equiv 1(\bmod n)$. Because of $a m \equiv s(\bmod n)$ we get the following congruences.

$$
\begin{aligned}
x_{i+1} & \equiv x_{i}+\frac{k}{n} \quad(\bmod 1) \\
x_{i+a} & \equiv x_{i}+\frac{a k}{n} \quad(\bmod 1) \\
m x_{i+a} & \equiv m x_{i}+\frac{a m k}{n} \equiv m x_{i}+\frac{1}{n} \quad(\bmod 1) .
\end{aligned}
$$

In the same way we get $m y_{i+a} \equiv m y_{i}+\frac{1}{n}(\bmod 1)$. The height function is $f_{3}(t)=g(m t+\phi)$. Since the multiplication by $m$ is already done in the computation above, the heights of the $m x_{1+l \cdot a}$ and of the $m y_{1+l \cdot a}$ for $l=0, \ldots, n-1$ differ only by a constant, see the figure.

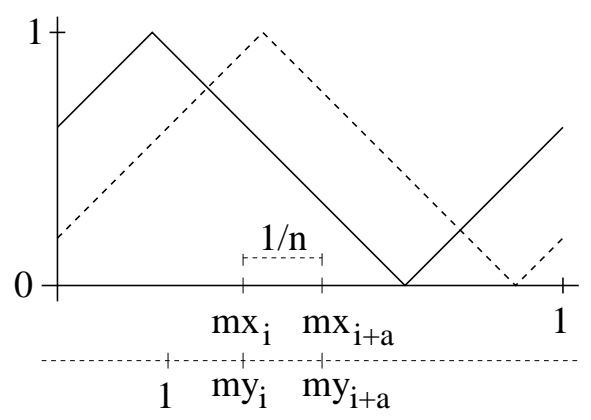

For even $n$ we choose for $b_{j}$ the uniquely determined index for which $g\left(m x_{b_{j}}+\phi\right)>$ $g\left(m y_{b_{j}}+\phi\right)$ and $g\left(m x_{b_{j}-a}+\phi\right)<g\left(m y_{b_{j}-a}+\phi\right)$. For odd $n$ we choose the $b_{j}$ which is at the beginning of the longer series of identical signs (these can be positive or negative, as stated in the theorem).

Notation: We define $v_{j}:=\left(\varepsilon_{1, j}, \ldots, \varepsilon_{n, j}\right) \in\{+1,-1\}^{n}$ for all $j \in\{1, \ldots, s-1\}$. In the following two theorems we identify rotated and mirrored braids.

Theorem 4.2 Let $\operatorname{gcd}(n, m)=1$. Then, given $v_{1} \in\{+1,-1\}^{n}$, for each $j \geq 2$ there are at most two possibilities for $v_{j}$, and they differ only by sign. 
Proof: Assume a phase $\phi$ gives the crossing-sign vector $v_{j}$ and the height diagram looks like the following (different cases for even and odd $n$ ).
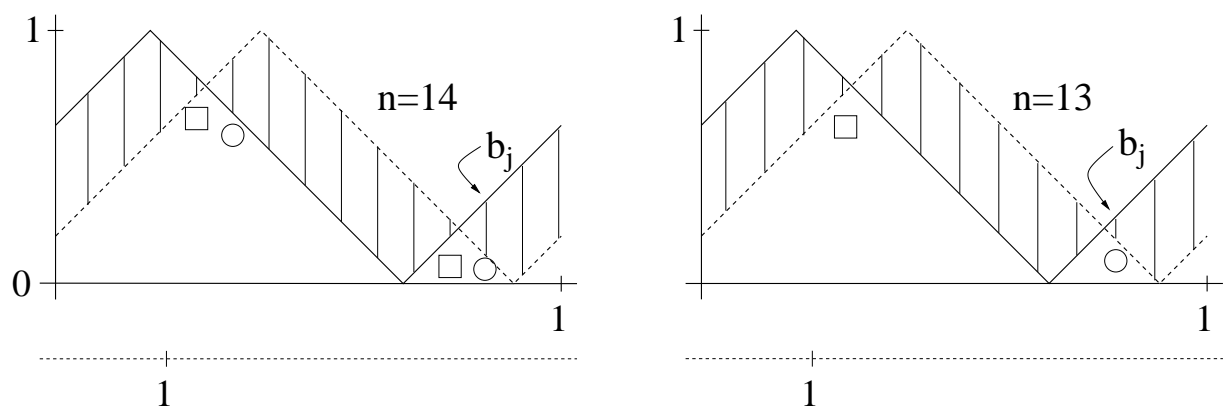

When we change the phase $\phi$ there are some crossings in danger to become singular and to be changed.

1. $n$ is even: Then, decreasing $\phi$ changes the pair of crossings $\left\{\left(b_{j}, j\right),\left(b_{j}+\frac{n}{2} a, j\right)\right\}$ and increasing $\phi$ changes the pair $\left\{\left(b_{j}-a, j\right),\left(b_{j}-a+\frac{n}{2} a, j\right)\right\}$. These two pairs are marked by circles and squares in the above figure. We call them the critical pairs (of crossings) of $v_{j}$.

2. $n$ is odd: In this case there is only the crossing $\left(b_{j}, j\right)$ which is changed for decreasing $\phi$, and for increasing $\phi$ the crossing $\left(b_{j}+\frac{n-1}{2} a, j\right)$. We call them the critical crossings of $v_{j}$.

Claim: The critical pairs (or critical crossings) of a crossing-sign vector determine it up to sign.

ProOF OF ClAIM: The critical pairs or critical crossings determine the beginning and end of the two series of identical crossing-signs because $n \geq 5$ if $s \geq 2$. If $n$ is even, the series have equal length $\frac{n}{2}$. If $n$ is odd the critical crossings belong to the longer series (of length $\frac{n+1}{2}$ ). In both cases there is only the choice of a global sign.

This fact yields the proposition for odd $j$ because the singular phases for $v_{1}$ coincide with those for $v_{j}$.

For odd $n$ and even $j$ a singularity at $(i, 1)$ implies a singularity at $\left(i+\frac{n-1}{2}, j\right)$, hence the critical crossings determine each other.

For even $n$ and even $j$ we use a different method: we place a maximum in the middle of a chord at $P$. Then exactly two crossings in $v_{1}$ are singular and the singular knot has a symmetry plane spanned by $P$ and the central axis. In $v_{j}$ there are exactly two indices $i$ with $\varepsilon_{i, j}=-\varepsilon_{i+a, j}$. Hence for $v_{j}$ there are only two possibilities $v_{j}^{\prime}$ and $v_{j}^{\prime \prime}=$ $-v_{j}^{\prime}$. Resolving the singularities by slightly moving the maximum away from $P$ gives two possibilities in each case but they are mirror images of each other. 


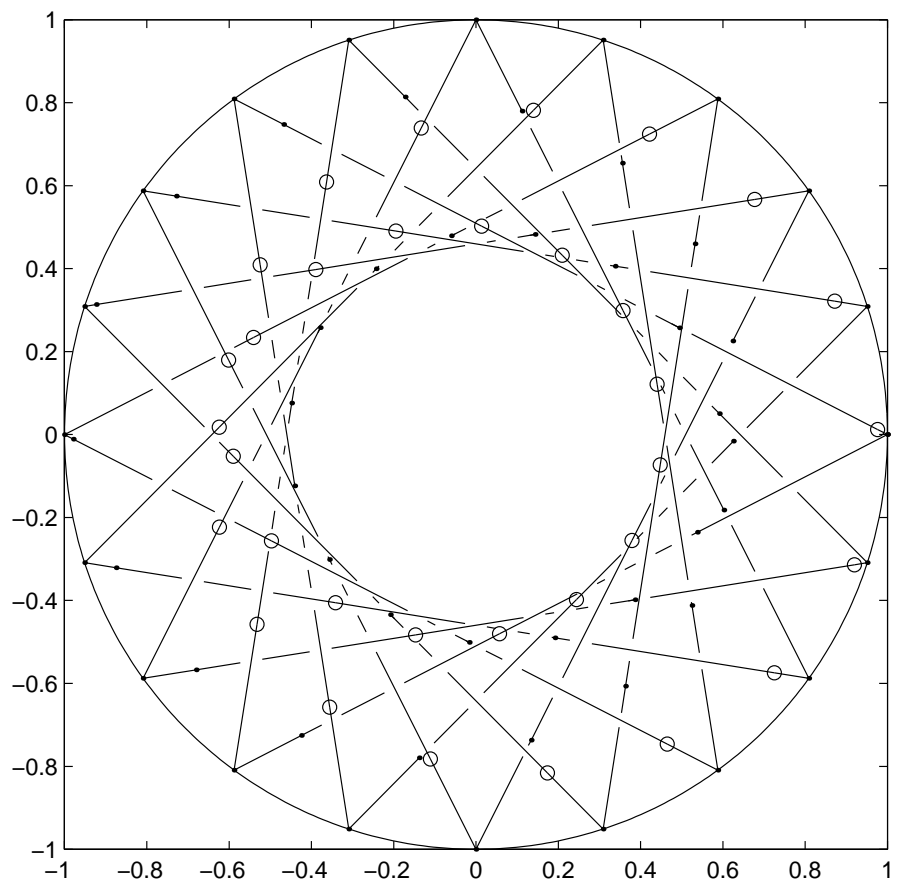

Figure 5: The cylinder knot $Z(7,20,33)$. It is ribbon and strongly positive amphicheiral.

Theorem 4.3 For given $s, n$ there are at most $(n+1) 2^{s-3}$ cylinder braids.

Proof: We count all possibilities for the $v_{j}$ for the different periods of the diagram. Let $d=\operatorname{gcd}(n, m)$ be the period of the diagram and $c=\frac{n}{d}$. Then for $c=1$ there are $2^{s-2}$ possibilities because we can choose $\varepsilon_{1,1}=1$ and there are two possibilities for every other $j$. (Following Remark 2.7, we can apply the Theorems 4.1 and 4.2 to the factor knots. The only exceptional case is $n / d=4$, because then every crossing belongs to a critical pair. We check this case directly by the method of placing a maximum in the middle of a chord or on a vertex.) For $c \geq 2$ there are $\frac{\varphi(c)}{2}$ possibilities to choose $a$, because $a$ and $-a$ give the same $v_{1}$. So there are at most $\frac{\varphi(c)}{2} \cdot 2^{s-2}$ possibilities for all $j$. Some care is needed in the case $c=2$. Here a braid with $v_{1}=v_{2}=(+1,-1)$ corresponds by a rotation around an axis in the diagram plane to a braid with $v_{1}=(+1,-1)$ and $v_{2}=(-1,+1)$. Hence there are $2^{s-3}=\frac{\varphi(2)}{2} \cdot 2^{s-2}$ possibilities. The sum over all $c$ is

$$
2^{s-2}+\sum_{\substack{c \mid n \\ c \geq 2}} \varphi(c) 2^{s-3}=2^{s-2}+(n-1) 2^{s-3}=(n+1) 2^{s-3} .
$$

\section{$5 \quad$ Rosette knots}

Rosette knots have been defined by Krötenheerdt [7] as a special class of alternating knots. These knots and the corresponding links can all be shown to be cylinder knots (or links), so we have quite a general class of examples. 
Definition 5.1 Let $k$ and $s$ be natural numbers. The closure of the $s$-string braid

$$
\left(\sigma_{1} \sigma_{2}^{-1} \sigma_{3} \sigma_{4}^{-1} \ldots \sigma_{s-1}^{(-1)^{s}}\right)^{k}
$$

is a link with $\operatorname{gcd}(s, k)$ components; we call it the rosette link $R_{s}^{k}$. In particular, if $k$ and $s$ are coprime, we call $R_{s}^{k}$ a rosette knot.

Remark 5.2 The rosette knots in the knot tables up to 10 crossings are the torus knots $t(2,2 n+1)=R_{2}^{2 n+1}$ for $n=1,2,3,4$, the figure-eight knot $4_{1}=R_{3}^{2}, 8_{18}=R_{3}^{4}, 10_{123}=R_{3}^{5}$ and $9_{40}=R_{4}^{3}$. For rosette links we have the well-known examples $R_{2}^{2}$ (Hopf link) and $R_{3}^{3}$ (Borromean rings).

Theorem 5.3 For $k \geq 2$ and $\operatorname{gcd}(k, s)=1$, the rosette knot $R_{s}^{k}$ is the cylinder knot $Z(s, k(s+1), k)$.

Proof: We start with the singular cylinder knot $K=Z(s, k(s+1), k, \phi)$ by placing a maximum on a vertex $P_{1}$. Because the billiard curve has period $k$ it is enough to look at the factor knot; we consider the slice with angle $\frac{2 \pi}{k}$ and deform the braid to a grid pattern for better visualization. We divide the height interval $[0,1]$ into layers of height $\frac{2}{s+1}$ (and for even $s$ one bottom layer of height $\frac{1}{s+1}$ ). Then on the symmetry planes there are singular crossings and the layering shows that the crossings in the first half of the slice are negative and in the second half they are positive (the convention is, that positive crossings correspond to the $\sigma_{i}^{-1}$ ).

See Figure 6 for $s=9$ as an example. Here we write $A, B, \ldots, E$ for the layers $\left[\frac{8}{10}, 1\right]$, $\left[\frac{6}{10}, \frac{8}{10}\right], \ldots,\left[0, \frac{2}{10}\right]$. For instance the chord $P_{1} P_{10}$ belongs to the layer $A, O_{10} P_{9}$ to $B$, hence $P_{1} P_{10}$ is above $O_{10} P_{9}$.

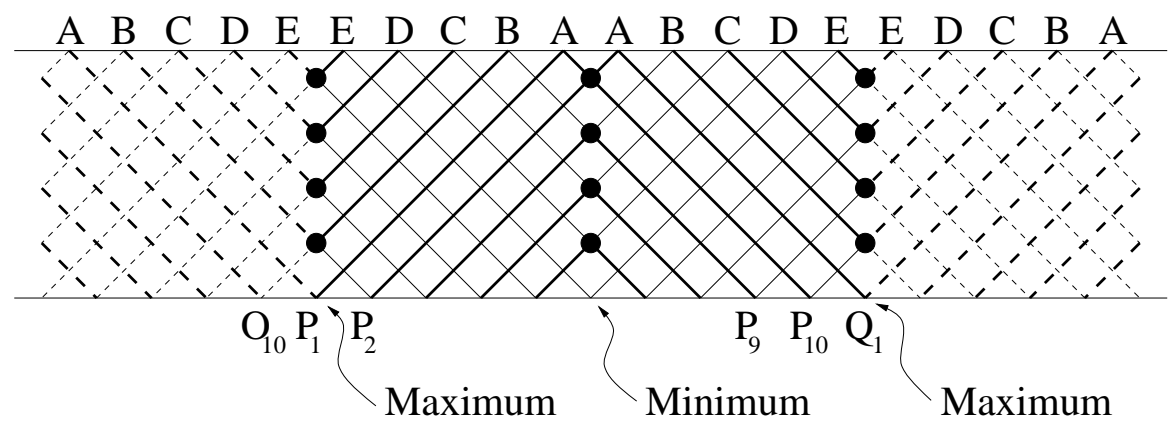

Figure 6: The braid (11) for $s=9$ with singularities on the symmetry planes

The next step is to push the maxima slightly to the left. This resolves the singularities to positive crossings at the planes at $P_{1}$ and $Q_{1}$ and to negative crossings in the central plane of the slice. Hence the factor knot has as a braid

$$
\left(\sigma_{1} \sigma_{3} \sigma_{5} \ldots \sigma_{s-2} \sigma_{2} \sigma_{4} \ldots \sigma_{s-1}\right)^{\frac{s+1}{2}}\left(\sigma_{1}^{-1} \sigma_{3}^{-1} \ldots \sigma_{s-2}^{-1} \sigma_{2}^{-1} \sigma_{4}^{-1} \ldots \sigma_{s-1}^{-1}\right)^{\frac{s+1}{2}}
$$


if $s$ is odd, and

$\left(\sigma_{1} \sigma_{3} \sigma_{5} \ldots \sigma_{s-1} \sigma_{2} \sigma_{4} \ldots \sigma_{s-2}\right)^{\frac{s}{2}}\left(\sigma_{1} \sigma_{3} \ldots \sigma_{s-1} \sigma_{2}^{-1} \sigma_{4}^{-1} \ldots \sigma_{s-2}^{-1}\right)\left(\sigma_{1}^{-1} \sigma_{3}^{-1} \ldots \sigma_{s-1}^{-1} \sigma_{2}^{-1} \sigma_{4}^{-1} \ldots \sigma_{s-2}^{-1}\right)^{\frac{s}{2}}$

if $s$ is even.

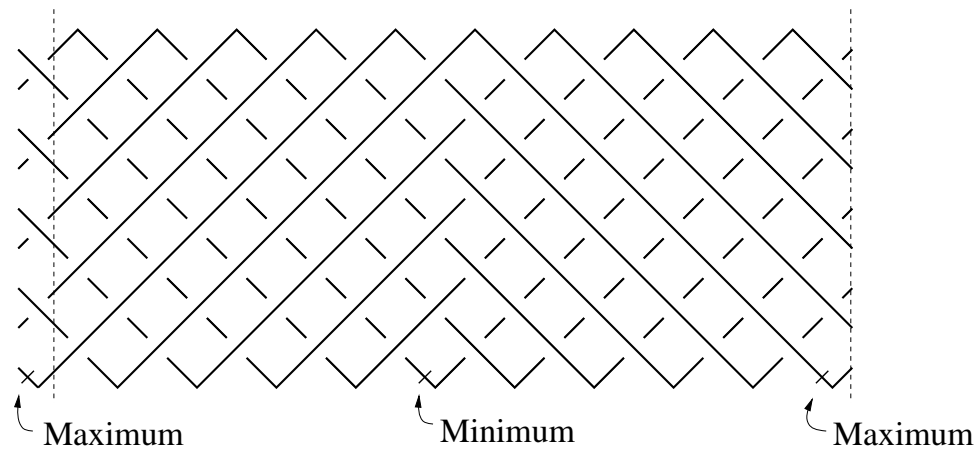

Figure 7: The braid (1) for $s=9$

We show by induction on $s$ that (1) is exactly the braid

$$
\sigma_{1} \sigma_{3} \sigma_{5} \ldots \sigma_{s-2} \sigma_{2}^{-1} \sigma_{4}^{-1} \ldots \sigma_{s-1}^{-1} \text {. }
$$

The induction step works as follows: In the braid (1), we observe that there is one string running above all the others and one running beneath, see Figure 0 0 . Hence we may pull out these two which leave only one negative crossing at the beginning and one positive at the end; the part in between then matches our induction hypothesis if we rotate it around the horizontal axis $P_{1} Q_{1}$ with an angle of $\pi$, and the proposition follows. By a similar argument we see that (2) is

$$
\sigma_{1} \sigma_{3} \sigma_{5} \ldots \sigma_{s-1} \sigma_{2}^{-1} \sigma_{4}^{-1} \ldots \sigma_{s-2}^{-1} .
$$

The reader is invited to prove that both braids are conjugate to

$$
\sigma_{1} \sigma_{2}^{-1} \sigma_{3} \sigma_{4}^{-1} \ldots \sigma_{s-1}^{(-1)^{s}}
$$

In the whole cylinder, we have $k$ times this braid; hence the proof is complete.

Remark 5.4 If $\operatorname{gcd}(k, s)>1$, then the construction yields the desired billiard link in the cylinder, but we have to assume that the vertices in the projection are equidistant, and for every component the maxima are at the right positions; in this case we cannot apply Lemma 2.3.

From [7] and [10] we already know that $R_{s}^{k}$ is amphicheiral if and only if $s$ is odd. Together with Lemma 2.6 we sharpen this condition:

Corollary 5.5 If both $k$ and $s$ are odd then $R_{s}^{k}$ is strongly positive amphicheiral. 
Conjecture 5.6 Any generalized rosette knot, i.e. the closure of

$$
\left(\sigma_{1}^{\varepsilon_{1}} \sigma_{2}^{\varepsilon_{2}} \ldots \sigma_{s-1}^{\varepsilon_{s-1}}\right)^{k}
$$

with $\varepsilon_{i} \in\{ \pm 1\}$, is a cylinder knot.

These knots are periodic with period $k$, linking number $\pm s$ and trivial factor knots. Hence they match the conditions of Theorem 3.3. For example all torus knots are generalized rosette knots.

We mention that besides rosette knots the following knots with low crossing numbers are cylinder knots: $9_{46}, 9_{47}, 10_{155}, 3_{1} \sharp 3_{1}^{*}, 5_{1} \sharp 5_{1}^{*}, 7_{1} \sharp 7_{1}^{*}$.

Acknowledgements: We thank D. Zagier for his help proving Lemma 2.4 and M. Veve for the graphics in Figures 6 and 5 .

\section{References}

[1] Bogle, M.G.V./Hearst, J.E./Jones, V.F.R./Stoilov, L.: Lissajous knots, J. Knot Theory and Ramifications 3, no. 2, 121-140 (1994).

[2] Fomenko, A.T.: Visual geometry and topology, Springer Verlag (1994).

[3] Galperin, G.A./Zemlyakov, A.N.: Mathematical billiard (in Russian), Kvant 77, Moscow (1990).

[4] Jones, V.F.R/Przytycki, J.H.: Lissajous knots and billiard knots, Banach Center Publications 42, 145-163 (1998).

[5] Kawauchi, A.: A Survey of Knot Theory, Birkhäuser Verlag (1996).

[6] Kinoshita,S./Terasaka, H.: On unions of knots, Osaka Math. Journal 9, 131-153 (1957).

[7] Krötenheerdt, O.: Über einen speziellen Typ alternierender Knoten, Math. Ann. 153, 270-284 (1964).

[8] Lamm, C.: There are infinitely many Lissajous knots, Manuscripta Math. 93, 29-37 (1997).

[9] Lamm, C.: Symmetric unions and ribbon knots, preprint (1998).

[10] Murasugi, K.: Remarks on rosette knots, Math. Ann. 158, 290-292 (1965).

[11] Obermeyer, D.: Billard-Knoten und Zylinderknoten, Diplomarbeit, Bonn (1998).

[12] Wang, K.: On a Theorem of S. Chowla, J. of Number Theory 15, 1-4 (1982).

[13] Washington, L.C.: Introduction to Cyclotomic Fields, GTM 83, Springer Verlag (1997). 